\title{
Health-Related Physical Fitness in Female Models
}

\author{
Salime Donida Chedid Lisboa', Rodrigo Sudatti Delevatti1,2, Ana Carolina Kanitz ${ }^{1,3}$, \\ Thais Reichert1, Cláudia Gomes Bracht1, Alexandra Ferreira Vieira1, \\ Luiz Fernando Martins Kruel ${ }^{1}$ \\ ${ }^{1}$ Universidade Federal do Rio Grande do Sul, Porto Alegre, Brazil \\ ${ }^{2}$ Faculdade Sogipa de Educação Física, Porto Alegre, Brazil \\ ${ }^{3}$ Universidade Federal de Uberlândia, Uberlândia, Brazil \\ Email: sa.lisboa@hotmail.com
}

Received 25 November 2015; accepted 26 January 2016; published 29 January 2016

Copyright (C) 2016 by authors and Scientific Research Publishing Inc.

This work is licensed under the Creative Commons Attribution International License (CC BY). http://creativecommons.org/licenses/by/4.0/

(c) (i) Open Access

\section{Abstract}

The model profession uses the appearance for the representation of products and brands via events. For some individuals that are included in this medium, plus a laboral activity, modeling becomes a lifestyle, the search for the status and work opportunities turn a dream for a thousands of children and teenagers because the profession has particulars experiences. To win this, many girls change physical and eating behaviors which are harmful to health. The objective of this study was to analyze the health-related physical fitness in female models comparing them with nonmodels. The study was conducted at the Caxias do Sul, Rio Grande do Sul, Brazil. Participated of the study female runway and commercial models bokered in Cast One Models, with age between 15 25 years old. The non-models were students from public and private schools or university students. The health-related physical fitness, the physical activity levels and dietary intake were assessed of all participants. The data were described as mean and standard deviation. For comparison between models and non-models was used $t$ independent test for variables normally distributed and U Mann-Whitney test for not normally distributed variables, adopting a level of significance $(\alpha)$ of 0.05 . It was found difference in total energy between model group and non-model (GM: $1509.78 \mathrm{kcal}, \mathrm{NM}$ : $2292.51 \mathrm{Kcal} ; \mathrm{p}=\mathbf{0 . 0 1 4}$ ). There were no differences between groups in the others variables analyzed $(p>0.05)$. In conclusion, the profession model seems not interfere in variables that make up the health-related physical fitness.

\section{Keywords}

Models, Health, Exercise 


\section{Introduction}

Adolescence is comprised between the age group from 10 and 19 years old [1], phase of life which is characterized by several changes in social, cultural and economic fields [2]. In this way, a proper nutrients ingestion and regular performance of physical activities become crucial, in order to obtain appropriate health levels. In this period, physical size and body shape become issues of concern for adolescents, because leanness has a big tendency for acceptance before society. Besides that, there are some professions with particular physical requirements, like athletes, ballet dancers and models, which are daily charged about the maintenance and/or weight reduction, which may become risk professions [3] [4].

The professional model term, in theory and practice, is a beauty model, a person who uses his/her appearance to represent products and brands, through publicity and events, what can many times become a lifestyle and/or professional career [5]. To be able to reach the status of being a model as a work career is a dream of thousands of children and adolescents, being these age groups in which the intensification of this dream increases, because this profession provides a glamour life to the ones who are successful and private experiences in each work done [6].

The required body measurements for models depend on the classification in which they are included. The classic categories are: photographic (commercial) and runaway model (fashion). Commercial models are characterized by performing mostly advertising works, like magazines, outdoors and television commercials. The attributions for this group, for both sexes, are to be photogenic along with well cared hair and skin. In relation to the body, measurements must be proportional, meeting the requirements of the contractor, depending on the required appeal for sealing of a particular product [7]. In regards to runaway models, they perform fashion parades and present season collections made by stylists, besides performing commercial works, like the photographic ones. The prerequisites to this kind of work are more rigorous, because the girls must begin with ages between 13 and 18, minimum height of $1.74 \mathrm{~m}$, hip measure $90 \mathrm{~cm}$ and waist about $60 \mathrm{~cm}$. Body weight must be reduced, approximately $20 \mathrm{~kg}$ less than the considered optimal for the age group. The other measures (bust and foot) vary according to body shape [8].

Discussions about models daily life conditions become increasingly delicate and explored by study fields like nutrition, psychology and physical education, since that besides being a profession with big turnovers due to contracts; it generates long working days, what can cause reductions in quality of life. Many sacrifices are adopted to reach the goals, because the girls start to give less importance to studies and the vast majority adopts eating and physical behaviors which are harmful to health. To the favoring of the leanness, adolescents from all over the world follow their own concepts of care, making them more susceptible to diseases and impairing their health in all the fields included in it [9].

The aforementioned state of health is not only characterized by a state of absence of diseases but as a general state of balance in different aspects as psychological, biological, emotional, social, mental and intellectual, thus enabling a well-being sensation that will be along with improvements in physical fitness of the individual and his/her relation to health, because physical activities practice is determined by physical fitness and health levels [10]. Guedes [11] defines physical fitness as a "the dynamic state of energy and vitality that permits to each person not only the performance of daily tasks, the active occupations of leisure time and to deal with unexpected emergencies without excessive fatigue, but also to avoid the appearance of hypokinetic functions, while operating at the peak of the intellectual capacity and feeling the enjoyment of living”. To obtain this, it is necessary to maintain the continuous practice of physical exercises and the search for the maintenance of physical fitness levels and its components when related to health (cardiorespiratory capacity, muscle strength/resistance and flexibility) [12].

Physical inactivity for lack of time and lack of stimulus along with bad eating habits, for care with appearance, are crucial points for the accomplishment of this work, because isolated evaluation of body composition, nutritional state and quality of life of adolescent models have already been found in the literature. However, we could not find studies that relate these variables in a same study, in adolescent and young adult models, because even with the advancement of exercise sciences, the importance of health-related physical fitness and the relation between exercise practice to quality of life in models are still little explored outcomes.

Therefore, the objective of the present study was to evaluate the health-related physical fitness, the physical activity levels and dietary intake in female models in comparison with non-models. 


\section{Methods}

\subsection{Subjects}

The sample of the study was composed by runaway and/or commercial female models between the ages of 15 and 25 from Cast One Models agency from the city of Caxias do Sul, Rio Grande do Sul, Brazil and also by adolescent and young adult students from public/private schools and universities from Caxias do Sul, Rio Grande do Sul, Brazil. The sample of the study was selected by convenience, being made contact with 16 models from Cast One Models agency, in which 10 accepted to participate in the study. After the selection of the models, a total of 8 non model girls were invited to participate in the study, of which 8 accepted it and were part of the study. These girls were also selected by convenience, in compliance with the same age groups and home city of the models, only avoiding the inclusion of obese girls, by believing that this would generate a huge comparative bias, once obesity is associated with physical activity level and physical fitness.

\subsection{Procedures}

The test protocols were performed at Estação Saúde gym in Caxias do Sul, Rio Grande do Sul, Brazil. 3 days were used for data collection, being the first day designated to the signature of a free and informed consent form, completing of an anamnetic record, body composition evaluation, filling of questionnaires about physical activity level, delivery and explanation of dietary records and familiarization with physical fitness tests (mensuration of peak oxygen consumption $\left(\mathrm{VO}_{2 \text { peak }}\right)$ ), flexibility, maximum dynamic muscle strength (1RM) of knee extensors and elbow flexors, resistant strength (muscular endurance) of knee extensors and elbow flexors). On the second day, a second familiarization with $1 \mathrm{RM}$ and muscular endurance tests was made and the cardiorrespiratory and flexibility tests were performed. On the third day, the 1RM and muscular endurance tests were performed, finalizing the experimental procedures. The reading and the signature of the consent form were made individually (for girls over the age of 18 years) and with the company of those responsible for them (for girls under the age of 18). An assent form was also filled to secure agreement between the study and the locals of the subjects' recruitment. In all the procedures, there were between two and three physical education professionals performing the aforementioned procedures.

Individuals attended to evaluation location according to the schedule, with two-piece suits. Firstly, height (HEI) and body weight (BW) measurements were made. With these values, their body mass index (BMI) was calculated, using the formula body weight $(\mathrm{Kg}) / \mathrm{height}^{2}(\mathrm{~m})$. After this, the measurements of the seven skinfolds were made: triceps, subscapularis, supra-iliac, abdominal, leg, middle axillary line and thigh. Based on the obtained values, body density was estimated utilizing the equation of the seven skinfolds proposed by Jackson et al. [13] for women.

For the evaluation of cardiorrespiratory fitness, a treadmill of Olympikus brand was utilized, with resolution of speed and inclination of $0.1 \mathrm{Km} \cdot \mathrm{h}^{-1}$ e $1 \%$, respectively, portable gas analyzer of mixing box type VO2000, INBRAMED brand, pneumotachograph, ranging from 2 to $225 \mathrm{~L} \cdot \mathrm{min}^{-1}$ for low, medium and high flows, neoprene mask and a heart rate monitor FT1 model, POLAR brand. Study subjects performed a familiarization session with the treadmill, the neoprene mask and the heart rate monitor that would be further used in the test. The maximum test on the treadmill was performed for the determination of the peak oxygen consumption $\left(\mathrm{VO}_{2 \text { peak }}\right)$ of the samples. For the accomplishment, individuals were instructed to stand with feet apart on the treadmill, where the neoprene mask would be put. To start the test, the respiratory exchange ratio (RER) should be under 0.85 . We utilized a protocol that consisted of an initial speed of $4 \mathrm{~km} / \mathrm{h}$ during two minutes, with increments of 1 $\mathrm{km} / \mathrm{h}$ at each two minutes, with fixed inclination (1\%). This protocol test was created for the conducting of the present study, aiming that the models reached the maximum effort without big increments in the inclination of the treadmill. Heart rate was recorded at each 10 seconds and the exertion perception was also registered at the end of each stage of the test. The test was conducted until voluntary exhaustion (signalized by manual gests). The evaluation was considered valid when one of the following criteria was reached at the end of the test [14]: 1) obtaining of the estimated $\mathrm{HR}_{\max }$ (220-age); 2) occurrence of a plateau in the $\mathrm{VO}_{2}$ with the increased velocity of the treadmill; 3) obtaining of a RER higher than 1.1; 4) perceived exertion higher than 17 (very intense-Borg's Scale of RPE).

The measurement of the maximal dynamic strength was obtained after a familiarization period of two sessions, with 1 RM tests for knee extension and elbow flexion. In these tests, individuals executed one repetition in each 
proposed exercise supporting the maximal possible load. The execution rhythm was controlled by a metronome (QUARTZ brand), being 1.5 seconds for the concentric phase and 1.5 seconds for the eccentric phase. Previously to the test, the girls performed a warm-up period ( 5 minutes) in cycle ergometer during five minutes, and after this an initial load was selected, in which the participants wouldn't be able to perform more than 10 maximum repetitions. After this series, the load was being adjusted with the corresponding of the 1RM through the values proposed by Lombardi [15] until the girls reached the maximal load. Up to 5 attempts were made, with an interval of 5 minutes between each one.

For the conduction of the dynamic muscular resistance test a load corresponding to $60 \%$ of $1 \mathrm{RM}$ of the knee extension and elbow flexor was utilized. For these tests, the individual should perform the maximal number of repetitions possible. The execution rhythm was controlled by a metronome, being 1.5 seconds designated for the concentric phase and 1.5 seconds for the eccentric phase.

The determination of the flexibility was made through a test utilizing the Wells Bench, which is in box format with the dimensions $30.5 \times 30.5 \times 30.5 \mathrm{~cm}$, containing in its upper flat a wooden board. The fixed scale is graded 1 by $1 \mathrm{~cm}$. The test was intended to measure the degree of flexibility of the hip, back and posterior muscles of the lower members. The evaluated individual was barefoot and assumed a sitting position, facing the equipment with the soles of the feet flat on the Wells Bench, with the knees fully extended. The arms were extended on the box surface with the hands overlapped one on another. The individual extended her body forward along the fixed scale, trying to reach the greatest possible distance. Three attempts were made and that for each one of them, the distance reached in the scale was maintained for at least 1 second long. The greatest value reached after all the attempts was considered [16].

The International Physical Activity Questionnaire (IPAQ) short version was utilized as indicator of the physical activity level of the study subjects, which investigates the physical activity level of the responders on the last seven days. This questionnaire consists of questions regarding the frequency of rigorous or moderate physical activity and walking activities performed in the last week by the responder. The present questionnaire can be classified categorically and/or in a continuous way, being estimated the metabolic units (METS) spent weekly (Met.min) in walking, moderate and vigorous physical activity, and the sum of these three conditions [17].

The feeding control was performed through the application of a feeding record of three days. The recording procedure was made through the following manner: each model registered three days of the week, non-consecutive, being two business days (typical days) and one weekend day (atypical day). The feeding records were filled during the collection period. The meals were described with the schedules, the quantities in household measures, and, when possible, the brand of the feeding products. After the filling of the records made by the girls, all of the notes were checked by a trained researcher, so that no doubt would happen in regarding to the described records. The feeding records were thereafter calculated with the aid of the Nutrition Software DietWin Professional (Brubins, CAS, Brazil) in order to quantify the content of the consumed food by the models and non models.

\subsection{Statistical Analysis}

As descriptive statistics the mean and standard deviation values were used for normally distributed continuous variables and the median and interquartile range values were used for continuous variables that were not normally distributed. Categorical variables were presented by the sample $n$. The normality and homogeneity of the data were evaluated by the Shapiro-Wilk and Levene tests, respectively. For the comparison between the variables of the models and the non models it was used the independent t-test for normally distributed variables and the Mann-Whitney U test for variables that were not normally distributed. When comparing categorical variables, we used Fisher's exact test. It was adopted a 5\% significance level. All analyzes were performed using SPSS program, version 20.0.

\section{Results}

\subsection{Flow of Participants}

24 girls were contacted, 16 of them were models and eight were non models. A total of six subjects left the study (six of MG, three because of contact impossibility, one because of family problems, one refused to participate and one because of address change). The adherence of the tests for the MG was impaired during the collection 
sessions due to the big turnover and long working days that are required by the profession, which caused absence of some models in some of the proposed protocols. In this way, the final population of the study consisted of 18 subjects performing the tests protocols (MG; $n=10$ ) and $(N M ; n=8)$. In Figure 1, the flowchart of the individuals over the study can be visualized.

\subsection{Participants}

All the girls who proposed to participate in the study were born and created in the same region, frequenting similar places. In MG, all the models were contracted by the same agency, with patterned solicitations and charges for all of them. It is noteworthy to mention the great importance that the MG components gave to the collection of the data (a large number of the models performed the collection procedures in order to obtain more information about their own body and thus try to reach the measures imposed by the agency in a healthy manner). In the same way, the non models participated in the study in order to obtain information that would favor their daily performance.

\subsection{Sample Characterization}

The characterization of the final sample (analyzed subjects) is presented on Table 1.

\subsection{Health-Related Physical Fitness}

All of the components of health-related physical fitness did not show any difference between the groups ( $\mathrm{p}>$ 0.05). These analyses are demonstrated on Table 2.

\subsection{Physical Activity Level}

The results found for scores of physical activity levels are presented on Table 3 . The variables also did not show any difference between the groups $(\mathrm{p}>0.05)$.

\subsection{Dietary Intake}

The results related to food consumption are presented on Table 4. A significant difference was found only for the total energetic value.

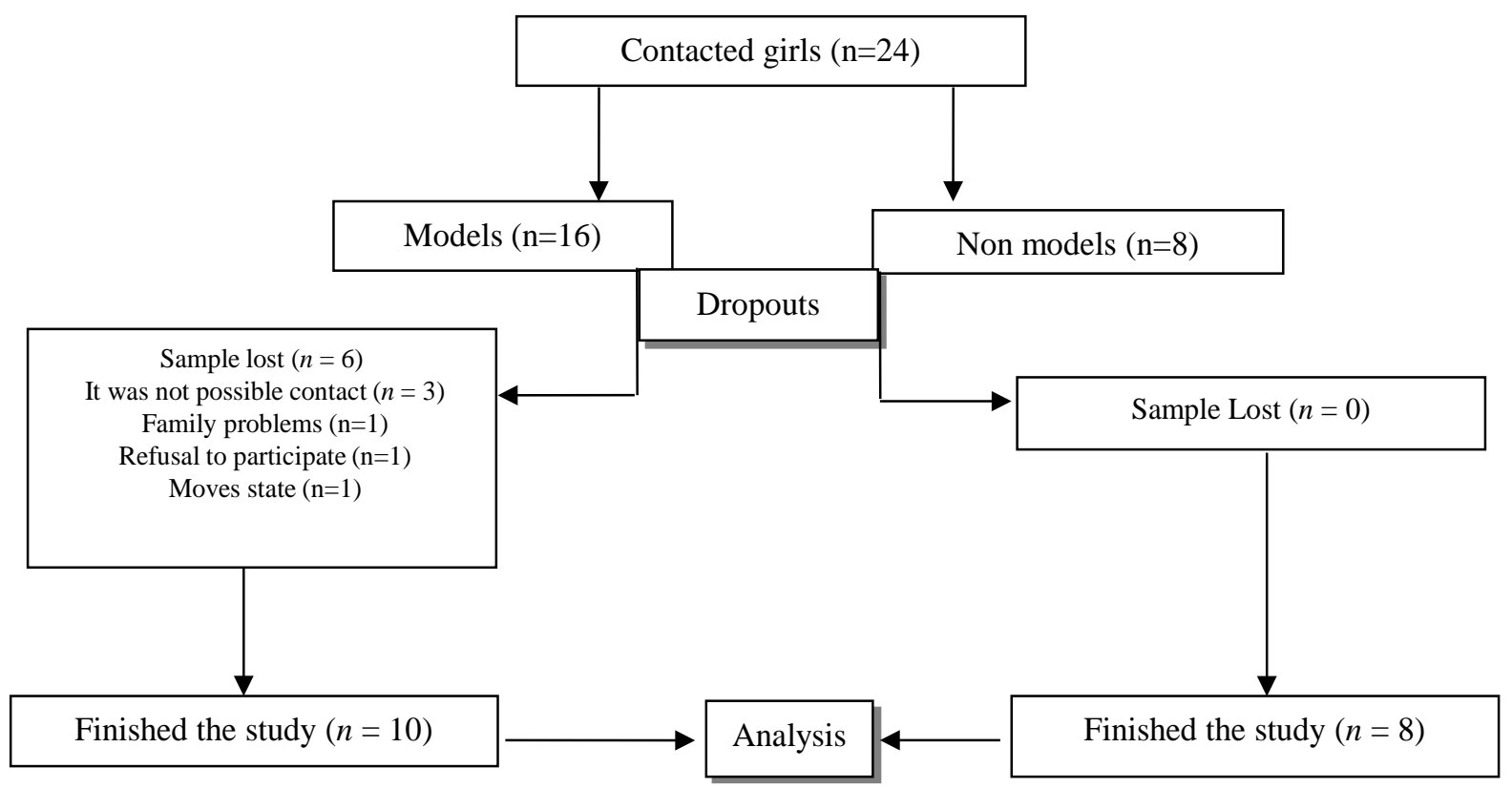

Figure 1. The flow of the participants. 
Table 1. Sample characterization.

\begin{tabular}{ccc}
\hline & MG $(\boldsymbol{n}=\mathbf{1 0})$ & NM $(\boldsymbol{n}=\mathbf{8})$ \\
\hline Age (years) & $20.5(16.2-21.7)$ & $21.5(20.2-23.0)$ \\
Career Duration (months) & $49.8 \pm 30.7$ & 2 \\
Nutritional monitoring & 2 & 4 \\
Psychic monitoring & 3 & 0 \\
Education & 0 & 0 \\
Fundamental Incomplete & 0 & 0 \\
Fundamental Complete & 3 & 1 \\
Medium Incomplete & 2 & 6 \\
Medium Complete & 5 & 1 \\
Superior Incomplete & 0 & 2 \\
Superior Complete & & \\
\hline
\end{tabular}

Age data are presented as median and interquartile range; Career duration is presented as mean $\pm \mathrm{SD}$; The others variables are presented by $\mathrm{n}$ of each group.

Table 2. Variables related to physical fitness and health in models (MG) and non models (NM).

\begin{tabular}{cccc}
\hline & MG $(\boldsymbol{n}=\mathbf{1 0})$ & NM $(\boldsymbol{n}=\mathbf{8})$ & p value \\
\hline VO$_{\text {2pico }}$ & $33.20(32.90-34.10)$ & $31.60(26.95-32.60)$ & $\mathrm{p}=0.073$ \\
1RM knee extension (Kg) & $70.00(65.00-72.50)$ & $65.00(55.00-72.50)$ & $\mathrm{p}=0.383$ \\
1RM bending elbows (Kg) & $16.00(16.00-19.00)$ & $18.00(16.00-18.00)$ & $\mathrm{p}=0.902$ \\
Resistant strength of knee extension (rep) & $9.00(8.00-11.00)$ & $12.00(10.00-12.50)$ & $\mathrm{p}=0.128$ \\
Resistant strength of bending elbows (rep) & $10.00(8.50-13.50)$ & $10.00(8.50-12.00)$ & $\mathrm{p}=0.902$ \\
Flexibility (cm) & $26.90 \pm 8.95$ & $26.25 \pm 4.77$ & $\mathrm{p}=0.856$ \\
Weight (kg) & $54.87 \pm 3.50$ & $53.38 \pm 5.02$ & $\mathrm{p}=0.484$ \\
Height (m) & $1.74(1.73-1.76)$ & $1.65(1.63-1.70)$ & $\mathrm{p}=0.002$ \\
BMI (kg/m $\mathbf{m}^{2}$ & $18.33 \pm 1.44$ & $19.19 \pm 1.57$ & $\mathrm{p}=0.239$ \\
Waist circumference (cm) & $67.50(66.00-68.70)$ & $67.25(65.12-70.62)$ & $\mathrm{p}=0.758$ \\
Waist/height ratio & $0.38(0.38-0.40)$ & $0.40(0.39-0.42)$ & $\mathrm{p}=0.252$ \\
Fat percentage & $16.37 \pm 3.04$ & $19.71 \pm 3.61$ & $\mathrm{p}=0.066$ \\
Percentage of lean mass & $83.63 \pm 3.04$ & $80.29 \pm 3.61$ & $\mathrm{p}=0.066$ \\
Fat mass (kg) & $9.03 \pm 2.01$ & $10.5 \pm 2.70$ & $\mathrm{p}=0.234$ \\
Lean mass (kg) & $45.83 \pm 2.49$ & $42.35 \pm 3.27$ & $\mathrm{p}=0.030$ \\
$\mathbf{\Sigma} 7 \mathbf{D C}$ & $86.40(78.15-94.40)$ & $98.50(87.42-114.60)$ & $\mathrm{p}=0.114$ \\
\hline
\end{tabular}

1RM: one repetition maximum; rep: repetitions. $\mathrm{VO}_{2 \text { peak }}$ data, $1 \mathrm{RM}$ knee extension, elbow flexion 1RM, resistant strength of knee extension, resistant strength of bending elbows, height, waist circumference and $57 \mathrm{DC}$ are presented as median and interquartile range (Mann-Whitney); The other variables are presented as mean $\pm \mathrm{SD}$ (independent $\mathrm{t}$ test). $\alpha: 0.05$.

Table 3. The level of physical activity rating by IPAQ questionnaire for Group models (MG) and non models (NM).

\begin{tabular}{|c|c|c|c|}
\hline & MG $(n=10)$ & $\mathrm{NM}(n=8)$ & p value \\
\hline Mets.min.walking & $297.00(148.50-569.25)$ & $264.00(185.63-305.25)$ & $\mathrm{p}=0.965$ \\
\hline Metsmin.PAmoderate & $360.00(0.00-1080.00)$ & $300.00(225.00-560.00)$ & $p=0.573$ \\
\hline Mets.min.PAvigorous & $360.00(0.00-1260.00)$ & $480.00(450.00-480.00)$ & $\mathrm{p}=0.897$ \\
\hline Mets.min.PAsum & $1306.50(572.25$ - 2219.25) & 1079.25 (957.00 - 1349.38) & $\mathrm{p}=0.897$ \\
\hline
\end{tabular}

PA: physical activity; Mets: metabolic unities. Data are presented as medians and interquartile ranges (Mann-Whitney). $\alpha$ : 0.05 . 
Table 4. Variable feed control through food records of three days to the model group (MG) and the group non models (NM) in percentage (\%) daily and grams (g) daily.

\begin{tabular}{|c|c|c|c|}
\hline & MG $(n=10)$ & NM $(n=8)$ & $p$ value \\
\hline Mean energetic value (kcal) & $1509.78 \pm 531.83$ & $2292.51 \pm 552.12$ & $\mathrm{p}=0.014$ \\
\hline Carbohydrates (g) & $202.20 \pm 80.61$ & $295.02 \pm 85.17$ & $\mathrm{p}=0.046$ \\
\hline Protein (g) & $63.17 \pm 20.98$ & $91.78 \pm 26.81$ & $\mathrm{p}=0.032$ \\
\hline Lipids (g) & $47.00 \pm 18.41$ & $79.00 \pm 20.34$ & $p=0.006$ \\
\hline Carbohydrates (\%) & $64.15 \pm 6.30$ & $62.97 \pm 6.59$ & $\mathrm{p}=0.727$ \\
\hline Protein (\%) & $20.72 \pm 4.96$ & $19.86 \pm 3.76$ & $\mathrm{p}=0.721$ \\
\hline Lipids (\%) & $15.12 \pm 3.10$ & $17.16 \pm 3.97$ & $p=0.270$ \\
\hline
\end{tabular}

Data are presented as mean \pm DE. $\alpha$ : 0.05. (Independent $t$ test).

\section{Discussion}

The main findings of the present study were that besides the supposed similarity found between models and non models, the girls who act like models showed some anthropometric differences (height and lean mass) and also a lower total energetic consumption. Rodrigues et al. [18] compared a group of models $(n=33)$ with non models ( $\mathrm{n}=33$ ), aged between 15 and 18, pairing them by age and BMI, analyzing body composition variables utilizing the plethysmography technique, resting metabolic rate (RMT) through indirect calorimetry and food consumption through three alternating days, and observed that many adolescents were showing their BMI under the recommended values for their age group, which corroborates the data found in our study, in which although any difference was found between the groups, in the MG the value was classified as low weight (18.3), while in NM the values correspond to the appropriate weight (19.2), according with the patterns of the Ministério da Saúde [19]. In this way, these body particularities of the MG also reflect on the fat percentages, because although only a statistical tendency exists ( $p=0.06$ ), the mean values of MG are $4 \%$ lower than those found in MG, showing tendency of the models to pursuit normative fat percentages values, to the point of being similar to the mean levels of fat (\%) of the non models.

Discussions regarding body dimensions are being increasingly made. By analyzing the models population, this discussion is related to the body that would be the one which contemplates the ideals of a body considered pattern. This is clearly exposed by Norton [20] who shows in this study the influence that a famous toy can cause in the construction of the body ideal of children and adolescents. By pointing some body measures of the Barbie doll, the researcher compared it to academic women (18 to 35 years old), to photographic and runaway models (seen as carriers of the ideal body) and also to anorexic girls. The results show that runaway models are leaner than academic women, and the anorexic girls, as was expected by disease questions, are leaner than all the other evaluated ones, and when the values of the doll are observed, a big difference is presented of body measures in comparison to the other groups of the study. The doll, which became an unconsciously imposed example for children, is criticized for being too lean, even when compared to the reference samples of the study, with similar measures found in less than 1 at each 100,000 adult women. The measures which showed more extreme values were neck circumference, wrist, waist, hip and waist hip ratio. This big restriction of body measures that used to be carried over from the doll to fashion runaways, and this way of thinking seem to be modified after the decade of 90 , and mainly, in the actual years due to rigid laws and rules that are being imposed to the models and their contractors, like the law against excessive leanness created by the National Assembly of France or agreements like the one made by the founder of the São Paulo Fashion Week, which vetoes the participation of people under 16 in the event. We believe in the efficacy of the laws and agreements through the results found in our variables, because there were no many differences found between models and non models, which can be indicating a new phase in the fashion world, in which the health is not necessarily detached from the model profession.

By analyzing the results obtained in the cardiorespiratory variables, there were no significant differences found in regards to the peak oxygen consumption $\left(\mathrm{VO}_{2 \text { peak }}\right)$ just as in the muscle strength and resistance and in flexibility. Possibly, no differences were found because of the similar number of exercise practitioners between the groups (MG: 6; NM: 5), which demonstrates that far beyond the chosen profession, the fact of carrying out a 
determined exercise modality seems to be fundamental for the fitness levels found, either cardiorrespiratory or neuromuscular ones. It demonstrates that much more than the profession, the adoption of a structured and supervises physical training can have a stronger implication in physical fitness than the labor activities of the individuals. Particularly in women, this is of fundamental importance, helping in the prevention of highly prevalent diseases in the female sex, as osteoporosis, that must be thought about since adolescence, a phase in which occurs the deposition of a big part (around 90\%) of the mineral bone content found in adult age [21].

In relation to physical activity levels, no significant differences were found between MG and NM, being both groups in scores corresponding to the sufficiently active classification (between 600 and 1499 mets.min.week), demonstrating that despite different labor occupations, the women of the present study had good physical activity levels, which is fundamental for health, because high levels of this outcome can reduce the incidence of many diseases, as well as mortality rates [22]. Our findings were satisfactory when compared to the findings of Hallal et al. [23] who studied the determinants of physical activities in the regional adolescence between the ages of 10 and 12, and obtained $58.2 \%$ of sedentary lifestyle scores, besides of, in the comparison between boys vs. girls, obtained scores of $40 \%$ and $67 \%$ respectively, pointing a likely higher prevalence of sedentary lifestyle for the female sex.

Moreover, the study of [18], aimed to evaluate the energetic expenditure of Brazilian models (33 models and non models aged between 15 and 18 years), found reports of up to $63.6 \%$ of the models and $60.6 \%$ of the non models performing regular physical activities, verifying that the models used to accomplish more activities when compared to control group (non models) $(5.0$ hours/week (IC 95\% $=3.9-4.9$ hours/week) versus. 2.0 hours/week (IC 95\% = 2.0 - 3.8 hours/week; $\mathrm{p}<0.01$ ). Even that this is a different unit for evaluation, our results corroborate to the aforementioned, because even without significant statistical differences, the values found in the models are shown to be more expressive tan the ones found for the non models (1306.50; 572.25; 2219.25 mets.min versus. 1079.25; 957.00; 1349.38 mets.min; $\mathrm{p}=0.897$ ).

Regarding to food consumption, the mean energetic value (kcal) ingested by the models is found next to 782.73 less daily kcal when compared to the non models, this lower caloric ingestion can be explained by the need and exigency of maintenance and many times of the loss of body mass daily requested by the models agency. The lower energetic consumption value of MG (MG: $1509.78 \pm 531.83$; NM: $2292.51 \pm 552.12$ ) can even explain the big tendency of difference $(\mathrm{p}=0.066)$ between the groups in the $\% \mathrm{~F}$. It demonstrates that the food rigidity imposed in the model profession ends to be really adopted and has an impact not only in area measurements, as in perimeters and BMI, but also in the accumulation of body fat. The difference between models and non models in the food consumption corroborates to the findings of Rodrigues et al. [24], that found significant differences in the caloric ingestion of models and non models (1480.93 kcal/day \pm 582.95 versus 1973.00 $\mathrm{kcal} /$ day \pm 557.63 respectively, $(\mathrm{p}=0.001$ ), showing that this difference does not seem to be an isolated fact, but a real difference of the profession in question when compared to general population.

Analyzing the daily percentages indicated by the recommendations of nutrition for adolescents [25], both MG and NM are ingesting above the recommended quantities of carbohydrates suggested for adolescents, that is between $55 \%$ to $60 \%$ (MG: $64.15 \% \pm 6.30 \%$; NM: $62.97 \% \pm 6.59 \%$ ). The same case happens for proteins, which has as suggested intake of $12 \%$ to $15 \%$ (MG: $20.72 \% \pm 4.96 \%$; NM: $19.86 \% \pm 3.76 \%$ ) and for lipids, with recommendations of 30\% (MG: $15.12 \% \pm 3.10 \%$; NM: $17.16 \% \pm 3.97 \%$ ).

It must be also noted that many girls who act as models are young and unprepared in questions of life, which causes the incorrect ingestion of foods (instant foods), being this action performed in order to gain time, contributing to a feeding considered not to be healthy, a worrying fact due to the evaluated age group and still, questions of altered ingestions, such as less fat than the recommended quantity, can implicate in injuries to women health in the future, because female hormones like estrogen and progesterone are synthesized based on fat [26].

As limitations of the present study, we highlight the reduced sample size, which is only representative of a profile of models and non models. Due to that, one should exercise caution in the interpretation of the results, being necessary to enlarge the observation of the health-related physical fitness, of the physical activity levels and of the nutritional profile in models of different levels, regions and ages.

\section{Conclusions}

Based on the results found in our study, we observed that the model profession can result in some anthropometric differences like greater height and lean mass, in addition to a lower energetic consumption. The comparison 
between models and non models showed a big similarity between the groups in aspects related to health-related physical fitness and physical activity levels. The findings of the study point that the model profession does not interfere in questions of health and its relation to physical fitness.

However, even reaching satisfactory results, we believe that this population has the need to be closely accompanied in all the ambits of life, due to the age group (adolescence and beginning of adult life) be a period of formation and consolidation of many questions of life and also because of the physical and psychological particularities that these adolescents are daily affected by, in order to avoid future problems in the health of these girls.

\section{References}

[1] The WHOQOL Group (1995) The World Health Organization Quality of Life Assessment (WHOQOL): Position Paper from the World Health Organization. Social Science \& Medicine, 41, 1403-1410. http://dx.doi.org/10.1016/0277-9536(95)00112-K

[2] Pires, L., Rodrigues, A.M., Fisberg, M., Costa, R.F. and Schoen, T.H. (2012) Quality of Life of Adolescent Professional Models. Psicologia: Teoria e Pesquisa, 28, 71-76. http://dx.doi.org/10.1590/S0102-37722012000100009

[3] Braggion, G.L., Matsudo, S.M.M. and Matsudo, V.K.R. (2000) Food Consumption, Physical Activity and Perception of the Body Appearance in Adolescents. Rev. Bras. Ciê. e Mov. Brasília, 8, 15-21.

[4] Rodrigues, A.M., Costa, R.F. and Fisberg, M. (2008) Anthropometric Characteristics of Candidates of a Selective Competition of a Big Agency from São Paulo. Centro de Estudos e Pesquisa Sanny.

[5] Pascolato, C. and Lacombe, M. (2003) How to Become a Successful Model-What It Is and How You See.

[6] Rodrigues, A.M., Cavalieri, M.C., Branco, L.M., Passos, M.A.Z., Cintra, I.P. and Fisberg, M. (2004) Anthropometric and Body Composition Profile of Adolecent Models. J. Brazilian Soc. Food Nutr., 27, 31-41.

[7] Libardi, M. (2004) Model Profession: In Search of Fame. SENAC SP, São Paulo.

[8] Castro, A.L. (2004) Body Worship: Identity of Life.

[9] Saikali, C.J., Soubhia, C.S., Scalfaro, B.M. and Cordás, T.A. (2004) Body Image in Eating Disorders. Revista de Psiquiatria Clinica, 31, 164-166. http://dx.doi.org/10.1590/S0101-60832004000400006

[10] Araújo, D.S.M.S. and Araújo, C.G.S. (2000) Physical Fitness, Health and Quality of Life Related to Health in Adults. Revista Brasileira de Medicina do Esporte, 6.

Bouchard, C., Shephard, R.J., Stephens, T., Sutton, J.R. and Mcpherson, B.D. (1990) Exercise, Fitness, and Health: The Consensus Statement. In: Bouchard, C., Shephard, R.J., Stephens, T., Sutton, J.R., McPherson, B.D., Eds., Exercise, Fitness, and Health: A Consensus of Current Knowledge, Champaign, Human Kinetics, 3-28.

[11] Guedes, D.P. (1996) Physical Activity, Physical Fitness and Health. In: Carvalho, T., Guedes, D.P. and Silva, J.G., Orgs., Orientações Básicas sobre Atividade Física e Saúde para Profissionais das Áreas de Educação e Saúde, Ministério da Saúde e Ministério da Educação e do Desporto, Brasília.

[12] Malina, R. (2007) Pediatric Fitness, Secular Trends and Geographic Variability. Tomkinson, G.R., Olds, T.S., Eds., Tarleton State University, Stephenville.

[13] Jackson, A.S., Pollock, M.L. and Ward, A. (1980) Generalized Equations for Predicting Body Density of Women. Medicine \& Science in Sports \& Exercise, 12, 175-182. http://dx.doi.org/10.1249/00005768-198023000-00009

[14] Howley, E.T., Basset Jr., D.R. and Welch, H.G. (1995) Criteria for Maximal Oxygen Uptake: Review and Commentary. Medicine \& Science in Sports \& Exercise, 27, 1292-1301. http://dx.doi.org/10.1249/00005768-199509000-00009

[15] Lombardi, V.P. (1989) Beginning Weight Training: The Safe and Effective Way. Brown \& Benchmark Pub, Dubuque.

[16] Charro, M.A., Bacurau, R.F.P., Navarro, F. and Pontes Jr., F.L. (2010) Physical Evaluation Manual. Phorte, São Paulo.

[17] Matsudo, S., Araújo, T., Matsudo, V., Andrade, D., Andrade, E., Oliveira, L. and Braggion, G. (2001) International Questionnaire of Physical Activity (IQPA): Study of Validity and Reproducibility in Brazil. Revista Atividade Física \& Saúde, 6, 5-18.

[18] Rodrigues, A.M., Cintra, I.P., Santos, L.C., Mello, M.T., Tufik, S. and Fisberg, M. (2009) Body Composition, Energy Expenditure and Food Consumption in Brazilian Models. Revista Brasileira de Cineantropometria e Desempenho Humano, 11, 1-7.

[19] Ministério da Saúde (2004) Food and Nutritional Surveillance, Série A. Standards and Manual Techniques.

[20] Norton, K.I., Olds, T.S., Olive, S. and Dank, S. (1996) Ken and Barbie at Life Size. Sex Roles, 34, 287-294. http://dx.doi.org/10.1007/bf01544300

[21] Bouchard, C., Shephard, R.J. and Stephens, T. (Eds.) (1994) Physical Activity, Fitness, and Health. In: Bouchard, C., 
Shephard, R.J. and Stephens, T., Eds., International Proceedings and Consensus Statement, Human Kinetics Publishers, Champaign, 931-942. http://dx.doi.org/10.1249/00005768-199401000-00024

[22] Vina, J., Sanchis-Gomar, F., Martinez-Bello, V. and Gomez-Cabrera, M.C. (2012) Exercise Acts as a Drug; the Pharmacological Benefits of Exercise. British Journal of Pharmacology, 167, 1-12.

[23] Hallal, P.C., Bertoldi, A.D., Gonçalves, H. and Victora, C.G. (2006) Sedentary Lifestyle Prevalence and Associated Factors in Adolescents Aged between 10-12 Years. Cadernos de Saúde Pública, 22, 1277-1287. http://dx.doi.org/10.1590/S0102-311X2006000600017

[24] Rodrigues, A.M., Cintra, I.P., Santos, L.C., Martini, L.A., Mello, M.T. and Fisberg, M. (2010) Adolescent Runaway Models: How Is the Food Consumption of This Group? Revista Paulista de Pediatria, 28, 326-332. http://dx.doi.org/10.1590/S0103-05822010000400007

[25] Gianinni, D.T. (2007) Nutritional Recommendations of the Adolescent: Adolescence and Health. Vol. 4.

[26] Mottini, D.U., Cadore, E.L. and Kruel, L.F.M. (2008) Exercise Effects on the Bone Mineral Density. Motriz: Revista de Educação Física, 14, 85-95. 